\title{
Papers
}

\section{Qualitative analysis of psychosocial impact of diagnosis of Chlamydia trachomatis: implications for screening}

\author{
Barbara Duncan, Graham Hart, Anne Scoular, Alison Bigrigg
}

\begin{abstract}
Objectives To investigate the psychosocial impact for women of a diagnosis of Chlamydia trachomatis and discuss the implications for the proposed UK chlamydia screening programme.

Design Qualitative study with semistructured interviews. Interview transcripts analysed to identify recurrent themes.

Participants Seventeen women with a current or recent diagnosis of chlamydia.

Setting A family planning clinic and a genitourinary medicine clinic in Glasgow.

Results Three themes were identified: perceptions of stigma associated with sexually transmitted infection, uncertainty about reproductive health after diagnosis, and anxieties regarding partner's reaction to diagnosis. Most women had not previously perceived sexually transmitted infections as personally relevant; this was a function of stereotypical beliefs about who was "at risk" of sexually transmitted infection. These beliefs were pervasive and negatively affected reactions to diagnosis and produced anxiety about disclosure of the condition to others (particularly sexual partners) and future reproductive morbidity. This anxiety, given the uncertain natural history of chlamydia, may prove difficult to dispel.

Conclusions There are three primary areas of concern for women after a diagnosis of chlamydia which need to be examined in the proposed screening programme. Information provided should normalise and destigmatise chlamydial infection and positively promote genitourinary medicine services. Support services should be available because notification of partner can cause anxiety. Uncertainty about future reproductive morbidity may be inevitable; staff providing screening will require guidance in providing advice under such conditions.
\end{abstract}

\section{Introduction}

The sexually transmitted infection Chlamydia trachomatis is a leading cause of reproductive morbidity in women, including pelvic inflammatory disease and infertility. ${ }^{1}$ Detection is difficult as it is largely symptomatic. Screening has been shown to reduce the prevalence of chlamydia in women ${ }^{2}$ and the incidence of pelvic inflammatory disease. ${ }^{3}$ Currently, on the recommendation of an expert advisory group to the chief medical officer, two pilot studies are being undertaken to assess the feasibility of implementing a screening programme in the United Kingdom. A major target group for screening would be young women attending primary care providers. ${ }^{4}$

Women being screened will need information and support and may experience psychosocial problems associated with screening. Because of the complex emotional investments and social taboos surrounding sexual relationships ${ }^{5}$ reactions to a diagnosis of a sexually transmitted infection are likely to be complex. Qualitative methods of research are appropriate for such complex issues. ${ }^{67}$ We used these methods to explore women's experiences of diagnosis of $C$ trachomatis and identify salient issues before the implementation of the pilot screening programmes. We investigated the psychosocial impact of diagnosis and discussed the implications of the results for the proposed national screening programme.

\section{Methods}

Participants and recruitment

We recruited women with a current or recent diagnosis of chlamydia who were currently attending either a genitourinary medicine clinic or a family planning clinic in central Glasgow. Women were either approached directly by the researcher (BD) after their consultation or treatment, or both, and invited to take part, or approached by clinic staff, who obtained written consent from women to release contact details to the researcher. Written consent was obtained from all women before interview, and ethical approval was given by both health trusts involved.

The sample comprised the first 17 women who agreed to participate (10 from the genitourinary medicine clinic and seven from the family planning clinic; response rate $62 \%$ ). We considered this sampling method appropriate because of the problems inherent in recruitment to studies such as this and time constraints. The mean time between treatment and interview was 11 weeks, with the exception of one woman who was awaiting the result of a recent chlamydia test and who had been diagnosed with chlamydia three years earlier. Women were aged between 18 and 29 years. Among women recruited in the genitourinary medicine clinic, eight out of 10 had been referred by other health providers. Women attending this clinic were also more likely than women attending the family

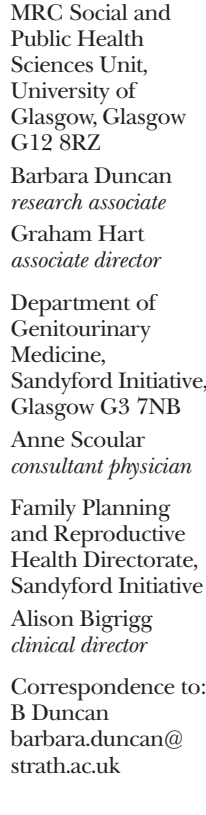

BMJ 2001;322:195-9

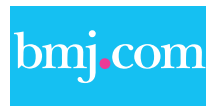

Details of the interview schedule can be found on the BMJ's website 


\section{Box 1 Details of participants}

Genitourinary medicine clinic

Reasons for attending clinic

Referred by: general practitioner (5), family planning clinic (2), gynaecology services after termination of pregnancy (1), sexual partner (2)

Employment status

Student (1), unemployed (1), financial management (2), administrative/clerical (5), sales assistant (1)

Relationship status

Cohabiting (1), regular relationship (6), single (3)

Presenting symptoms and comorbidity (when present) Abdominal pain (gonorrhoea) (1), genital warts (4), abdominal pain (pelvic inflammatory disease) and genital warts (1), irregular menstruation (1)

\section{Family planning clinic}

Reasons for attending clinic

Emergency contraception: requested sexual health screen (1), advised to have sexual health screen (1), referred by sexual partner (1), attended for sexual health screen (1), seeking treatment for symptoms related to sexually transmitted disease (1), insertion of intrauterine device (1), cervical smear (1)

Employment status

Student (4), financial management (1),

administrative/clerical (1), care worker (1)

Relationship status

Regular relationship (5), single (2)

Presenting symptoms and comorbidity (when present) Urinary symptoms (1)

planning clinic to report symptoms relating to sexually transmitted infection, although this could largely be attributed to the presence of comorbidity (see box 1).

\section{Interviews and analysis}

All interviews were conducted by $\mathrm{BD}$ in a clinic setting at the MRC Unit or in the women's homes. Interviews lasted between 40 minutes and two hours and were tape recorded and transcribed verbatim. We used a semistructured interview schedule, which explored women's experiences of being diagnosed with chlamydia. Questions were open ended, and we adopted a non-directive approach to encourage women to develop and elaborate their own narratives about their experiences (details of the interview schedule can be found on the $B M$ /s website).

Data were analysed with interpretative phenomenological analysis, ${ }^{8}$ which seeks to capture the meaning to the participant of the phenomenon under investigation. Individual transcripts were read repeatedly and then coded to identify emergent themes. Recurrent themes were then identified across transcripts; such themes reflect a shared understanding among participants of the phenomena under investigation. This was a dynamic process, with each transcript informing both the collection of further data and their subsequent analysis. ${ }^{9} \mathrm{BD}$ carried out the principal analysis. A subsample of transcripts was read independently by $\mathrm{GH}$ and emergent and recurrent themes discussed. The themes were agreed with AS and $\mathrm{AB}$.

\section{Results}

The themes that have implications for future screening programmes were perceptions of stigma associated with sexually transmitted infections, uncertainty about future reproductive health, and anxieties regarding male partners' attitudes to diagnosis.

\section{Stigma}

Before diagnosis most participants had perceived themselves as relatively invulnerable to infection. In the women's accounts, sexually transmitted infections were associated with stereotypical notions of contamination and delinquency. Participants distanced themselves from the "type" of person likely to contract a sexually transmitted infection (see box 2), which led them to believe that chlamydia and other sexually transmitted infections were not personally relevant. These stereotypes also affected expectations of the genitourinary medicine clinic, and initial reactions to referral were generally negative. While six women reported some knowledge of chlamydia before diagnosis, only two acknowledged any sense of personal vulnerability to infection. Of the eight women who had presented with

Box 2 Perceptions of stigma associated with sexually transmitted infections (place of attendance in parentheses)

Feelings on initially attending the clinics I was all very shy when I went in [to the clinic] I think. When you go you feel-well, I felt really dirty because, because it's an STD [sexually transmitted disease] basically and I thought people like me don't get these kind of things. (Respondent 2, family planning clinic)

Feelings of stigma associated with the genitourinary medicine clinic

In all honesty, like I always imagined that [the clinic was] like ... this is terrible, this is totally judgmental, but, like seedy, seedy people and people that are ... not prostitutes, I wouldn't go as far as to say that, but just a lot ... that sort of place, you know, like filthy men go and a lot of men sitting about. 'Cos I mean, I don't sleep about and that's why, like, why do I have to go to one of these places, you know. I guess you have like a preconceived idea about what it's gonna be like and it was totally fine. (Respondent 4, genitourinary medicine clinic)

Reaction to diagnosis

BD: How did you feel?

A: Absolutely gob smacked ... Totally shocked. BD: When they [clinic staff] said they thought there was something wrong, what did you think was wrong? A: I don't know. ... that way I was I knew there was something wrong but I suppose it was the kind of last thing that you think about.

BD: Had you been having any symptoms or anything? A: Uhuh, I'd been in like quite a bit of pain. (Respondent 1 , genitourinary medicine clinic)

Disclosing diagnosis to others

You don't phone up everyone and say I've got a sexually transmitted disease you know, I'm not feeling too good come round and see me, cheer me up ... From that point of view you feel very isolated because you can't really talk about it, I suppose you could but you know other people's reactions would put you off talking about it. (Respondent 3, genitourinary medicine clinic) 
symptoms associated with sexually transmitted infections, only four reported that, before attending a health provider, they had correctly attributed their symptoms to such an infection (one woman with urinary symptoms, one with abdominal pain, one with irregular menstruation, and one with genital warts).

For most women, therefore, receiving a diagnosis of chlamydia was a shock, and all women reported experiencing feelings that ranged from mild self disgust to distress. Women also expressed worry about disclosing their condition to others. Most did tell selected friends or family members, but none did so without some anxiety about negative reactions. Disclosure to others functioned as a mechanism for garnering social support, and feelings of isolation were reported by women who felt unable to tell members of their usual support network.

\section{Future reproductive health}

All of the women reported receiving verbal or written information, or both, and advice from clinic staff about chlamydial infection, treatment, and possible effects. Provision of such information varied; all attenders of genitourinary medicine clinics reported receiving information and advice from health advisors, a service not available to women attending the family planning clinic. When participants were asked about the content of such information, they most often recalled the possibility of infertility after infection (see box 3). This provoked a mixed reaction: relief that the infection had been diagnosed and treated but also anxiety about future reproductive morbidity. These anxieties were exacerbated by clinical uncertainty about the natural course of chlamydia and the difficulty of providing a prognosis in relation to reproductive effects. ${ }^{1}$ The largely asymptomatic nature of the infection (13 of the women reported no related symptoms before diagnosis) meant that many of the participants were unsure about the length of time that they had been infected. As most women believed that there was a positive association between duration of infection and probability of future infertility, this provided a further source of continuing anxiety.

\section{Box 3 Future reproductive health}

Assessing the probability of future infertility So when I got a letter [with the positive diagnosis] it was a shock. I was worried ... it was the fact that it can make you infertile and when I had been to the doctor and I had asked her about pelvic inflammatory disease-like do you know if you have got it, like is there anyway to know how advanced chlamydia has got? And she said no not really until you start trying to conceive and you can't conceive that is when you discover, and I thought God-is this going to be the unknown thing until I'm trying to have a baby. The fact that I knew when I had contracted it-I thought maybe that would maybe give a timescale or an indication of whether or not it was likely to have done any damage, but she couldn't say ... I just said, you know, do you know if you have got pelvic inflammatory disease, it was the nurse that I was speaking to at this point and she said ... not always ... So anyway I don't know ... the things that I was worried about she [the doctor] didn't seem to give me any answers on. (Respondent 4, family planning clinic)
Box 4 Anxieties about male partners' attitudes to diagnosis

Informing a current partner

It wasn't a very pleasant experience ... that I think what hurt him more was the fact that I hadn't told him [about a previous partner], [he was] just very shocked. I don't know, he didn't like the idea of me having slept with too many people. But I tried to explain what happened and he understood ... I felt bad, I still feel bad about it but I felt really bad that night and the following day I just couldn't think of anything else. I felt just guilt more than anything else, regret. I regretted it [previous partner] at the time and then the fact I've harmed him [current partner] as well just made it worse. (Respondent 1, family planning clinic)

\section{Informing a former partner}

Em ... I don't really know how to put it because we had been on bad terms he [former partner] had made life hell, you know and I had just been trying to extricate myself from it. So I hadn't really had any contact with him, but I just said I had been told I had this and like how long I have had it for and it was possible that it would cause infertility, and he shouted and bawled saying are you trying to say that I gave it to you ... So it was very tough to tell him but he was fine. (Respondent 4, family planning clinic)

Feeling unable to inform a former partner ... things had ended very badly ... and no, I couldn't have approached him on anything-for a cup of tea-never mind something like that, you know ... under the circumstances I did want to go back to previous partners and say, because I think that it's important that you do. And I had dreadful guilt, oh my goodness what if I've passed this on to somebody else and some other poor woman can't have children because I've not had the guts to go and say. (Respondent 7, family planning clinic)

\section{Anxieties about attitudes of male partners}

Participants' sexual relationships were mainly serially monogamous, with some women having sex with casual partners between relationships. For women with chlamydia, guidelines suggest that all partners in the past six months should be contacted. ${ }^{10}$ This relatively long period of possible infectivity meant that women often attributed the source of infection to a previous relationship. Diagnosis of a sexually transmitted infection introduced the possibility of a current partner's infidelity, a possibility that could be discounted if the source of infection was thought to be a previous relationship. Clinic staff encouraged this belief; there was wide variation in the reported likely duration of infection (from a few weeks to seven years). Given this variation, a plausible explanation is that health professionals were exploiting the uncertainty surrounding the natural course of chlamydia infection to mitigate the threat to the current relationship. Emphasis of the uncertainty about duration of infection may lessen fears about a partner's infidelity but could also increase anxiety about possible reproductive morbidity.

Despite the norm of serial monogamy among participants and, reportedly, their partners, disclosure was associated with fears of negative reactions by sexual partners. Feelings of guilt, regret, and "dirtiness" were also reported in this context. While disclosure did not seriously threaten the current, regular relationships of the women interviewed here, their fears of partners' 
reactions were real and a considerable source of worry (see box 4). Informing a previous partner was perceived as difficult, especially when that relationship had ended badly. Some women chose not to notify former partners; this decision could also have psychological costs for women, such as feelings of guilt at their perceived "irresponsibility."

\section{Discussion}

Our results identified three primary areas of concern after a diagnosis of chlamydia: the perceived stigma of sexually transmitted infections, worry about future reproductive health, and anxiety associated with notifying partners. Our results have several implications for the proposed screening programme. Firstly, information given to women before screening should seek to normalise and destigmatise chlamydial infection to reduce the negative psychological impact of a positive diagnosis. Secondly, although it was clear that the information given to women by staff served to lessen, if not eradicate, stigma, disclosure of the condition to others remained a source of anxiety (specifically, that others would react badly). This anxiety may be exacerbated if women feel unable to access their usual support network. Thus, support services should be available if required. Women attending a genitourinary medicine clinic highlighted the important role of health advisors in providing advice and reassurance. Given the uncertainties associated with chlamydial infection and that reassurance about one factor can increase anxiety about another, staff outside specialised services may require guidance in providing support to women diagnosed with infection. Finally the chief medical officer's report recommends that women with positive diagnoses should be referred to genitourinary medicine clinics for support and advice about telling partners. It acknowledges that some patients may not take up referral and that education is required to destigmatise genitourinary medicine services. ${ }^{4}$ The data reported here support this position. This is not simply a matter of partner notification; comorbidity is of concern, and those identified positive for chlamydia may require a full sexual health screen to ensure that other infections are diagnosed and managed appropriately. Genitourinary medicine clinics must be represented as accessible and nonjudgmental sexual health services.

Our data were not from women who had undergone chlamydia screening as part of a national screening programme, but these accounts can help inform our understanding of some of the possible reactions of women identified through such a programme to the news that they are infected with $C$ trachomatis. We do not make any claims regarding the generalisability of this exploratory study, but, given the lack of available research in this area, the data provide important insights. Some of our results echo those of other studies-for example, the stigma, ${ }^{112}$ and isolation $^{11}$ associated with a diagnosis of sexually transmitted infection and relatively low levels of knowledge of chlamydia. ${ }^{13}$ It is notable that only six of the women in the study (three who had attended the genitourinary medicine clinic and three who had attended the family planning clinic) had, before diagnosis, perceived themselves to be personally vulnerable to a sexually
What is already known on this topic

Little is known about the psychosocial implications of a diagnosis of chlamydia, which is an important issue in the context of the proposed UK chlamydia screening programme

\section{What this study adds}

Women are concerned about the perceived stigma of sexually transmitted infections, future reproductive health, and notifying partners

Messages accompanying screening should not imply that diagnosis and treatment will prevent infertility, and uncertainty about future reproductive morbidity may be an inevitable cost of screening

Information given to women before screening should seek to normalise and destigmatise chlamydial infection to reduce the negative psychosocial impact of a positive diagnosis

transmitted infection and had actively sought treatment for this reason. Thus, this sample of women is unlikely to differ substantially from women recruited to a national screening programme in terms of perceived risk of chlamydia, and their reactions to diagnosis (and to referral to a genitourinary medicine clinic) are likely to be comparable. A recent study of 20 women who had been screened for chlamydia in general practice yielded similar results. ${ }^{14}$

From the accounts of these women, a diagnosis of chlamydia triggered rather than allayed uncertainty about future reproductive morbidity. Current knowledge of the natural course of chlamydia is insufficient to provide complete reassurance for individual women about their future reproductive health. It is imperative that care is taken to ensure that women do not develop unrealistic expectations of chlamydia screening-for example, accompanying information should not inadvertently imply that diagnosis and treatment of chlamydia will, in itself, prevent infertility. Indeed, given the current state of knowledge about chlamydia, some uncertainty about future reproductive health may be an inevitable cost of screening for those with positive diagnoses; this should be made clear to women before participation.

The proposed chlamydia screening programme has the laudable public health aim of reducing the incidence, and possibly eradicating, a treatable sexually transmitted infection with potentially serious effects on reproductive health. Before the implementation of any new national screening programme, however, research is required to identify strategies to maximise the uptake of the service while minimising uncertainty and allaying anxiety associated with positive test results.

Contributors: GH had the original idea for the study, designed the study with $\mathrm{BD}$, and was involved in data analysis and drafting the paper. BD collected the data, carried out primary data analysis, wrote the first draft of the paper, and is guarantor. AS and $\mathrm{AB}$ assisted in design, interpretation of data, and drafting the paper.

Funding: UK Medical Research Council.

Competing interests: None declared. 
1 Cates W, Wasserheit JN. Genital chlamydial infections: epidemiology and reproductive sequelae. Am J Obstet Gynecol 1991;164:1771-81.

2 Centers for Disease Control. Chlamydia trachomatis genital infectionsUnited States 1995. JAMA 1997;277: 952-3.

3 Scholes D, Stergachis A, Heidrich FE, Andrilla H, Holmes KK, Stamm WE. Prevention of pelvic inflammatory diseases by screening for cervical chlamydial infection. N Engl J Med 1996;334:1352-66.

4 Chief Medical Officer. Main report of the CMO's expert advisory group on Chlamydia trachomatis. London: Department of Health, 1998.

5 Ainslie DC. AIDS, sexual ethics and the duty to warn. Hastings Center Report 1999;29:26-35.

6 Black N. Why we need qualitative research.J Epidemiol Community Health 1994;48:425-6.

7 Buston K, Parry-Jones W, Livingston M, Bogan A, Wood S. Qualitative research. Br J Psychiatry 1998;172:197-9.

8 Smith JA. Beyond the divide between cognition and discourse: using interpretative phenomenological analysis in health psychology. Psychol Health 1996;11:261-71
9 Flowers P, Smith JA, Sheeran P, Beail N. Health and romance: understanding unprotected sex in relationships between gay men. $\mathrm{Br} J$ Health Psychology 1997;1:73-86.

10 SIGN. Management of genital Chlamydia trachomatis infection: a national clinical guideline. Scottish Intercollegiate Guidelines Network: Edinburgh, 2000.

11 Holgate HS, Longman C. Some people's psychological experiences of attending a sexual health clinic and having a sexually transmitted infection. J R Soc Health 1998;118:94-6.

12 Redfern N, Hutchinson S. Women's experiences of repetitively contracting sexually transmitted diseases. Health Care for Women Int 1994;15:423-

13 Kellock DJ, Piercy H, Rogstad KF. Knowledge of Chlamydia trachomatis infection in genitourinary medicine clinic attenders. Sex Trans Inf 1999:75:36-40.

14 Santer M, Wyke S, Warner P. Screening women for chlamydia infection in general practice using urine testing. Edinburgh: Chief Scientist Office, 2000. (Accepted 28 September 2000)

\title{
Birth weight and cognitive function in the British 1946 birth cohort: longitudinal population based study
}

\author{
Marcus Richards, Rebecca Hardy, Diana Kuh, Michael E J Wadsworth
}

\begin{abstract}
Objective To examine the association between birth weight and cognitive function in the normal population.

Design A longitudinal, population based, birth cohort study.

Participants 3900 males and females born in 1946. Main outcome measures Cognitive function from childhood to middle life (measured at ages $8,11,15$, 26 , and 43 years).

Results Birth weight was significantly and positively associated with cognitive ability at age 8 (with an estimated standard deviation score of 0.44 (95\% confidence interval 0.28 to 0.59 )) between the lowest and highest birthweight categories after sex, father's social class, mother's education, and birth order were controlled for. This association was evident across the normal birthweight range $(>2.5 \mathrm{~kg})$ and so was not accounted for exclusively by low birth weight. The association was also observed at ages 11,15 , and 26, and weakly at age 43 , although these associations were dependent on the association at age 8 . Birth weight was also associated with education, with those of higher birth weight more likely to have achieved higher qualifications, and this effect was accounted for partly by cognitive function at age 8 .

Conclusions Birth weight was associated with cognitive ability at age 8 in the general population, and in the normal birthweight range. The effect at this age largely explains associations between birth weight and cognitive function at subsequent ages. Similarly, the association between birth weight and education was accounted for partly by earlier cognitive scores.
\end{abstract}

\section{Introduction}

Small size at birth is associated with a range of adverse health outcomes, ${ }^{1}$ including poor cognitive development, ${ }^{2}$ an effect that is largely unconfounded by features of the family environment, such as socioeconomic status and birth order. Although most cognitive studies focus on clinically low birthweight groups, confirmation of this association across the full birthweight range in the normal population is of particular interest, since this would imply that explanatory factors are similarly distributed in the general population. One early such population based study found that verbal reasoning scores at age 11 increased with increasing birth weight. ${ }^{3}$ This association was also detected in early adulthood, ${ }^{4}$ although not in later life.

We examined the association between birth weight and cognitive function in the normal population using data from the British 1946 birth cohort. A particular advantage of this cohort is the use of repeated cognitive assessments throughout life, beginning at age 8 years and extending to age 43 , thus allowing the investigation of relative change in cognitive function according to birth weight. To investigate an outcome with specific functional consequences, we also examined the association between birth weight and educational attainment.

\section{Methods}

\section{The 1946 birth cohort}

Participants were drawn from the Medical Research Council's national survey of health and development, a birth cohort study stratified by social class and initially consisting of 5362 people selected from all births that occurred in England, Scotland, and Wales during one week in March 1946. ${ }^{6}$ Information about sociodemographic factors and medical, cognitive, and psychological function has been obtained at intervals by interview and examination-most recently in 1989 at age 43 , when the sample size was 3262 ; the sample is regarded as representative of the UK population born legitimately and singly in the years immediately after the second world war.

\section{Cognitive measures and educational attainment} Various cognitive measures were used. ${ }^{8}{ }^{9}$ Children were tested at age 8 on reading comprehension, word pronunciation, vocabulary, and non-verbal reasoning;
MRC National Survey of Health and Development, University College London, London WC1E 6BT

Marcus Richards MRC scientist

Rebecca Hardy

MRC scientist

Diana Kuh

MRC senior scientist

Michael E J

Wadsworth

director

Correspondence to: M Richards, MRC

National Survey of

Health and

Development,

University College

London,

Department of

Epidemiology and

Public Health,

London

WC1E 6BT

m.richards@ucl.

ac.uk

BMJ 2001;322:199-203 